\title{
Maltrato Infantil: Riesgo de Caries Dental
}

\author{
Tomás Caudillo Joya, PhD \\ María del Pilar Adriano Anaya, PhD \\ Sergio Antonio Chavarría López, EE \\ Profesores de la Facultad de Estudios Superiores Zaragoza Universidad \\ Nacional Autónoma de México \\ Pilar Alejandra Caudillo Adriano, MDP \\ Práctica Privada
}

URL:http://dx.doi.org/10.19044/esj.2020.v16n6p43

\section{Resumen}

Objetivo: Realizar el Diagnóstico epidemiológico bucal en escolares del Modelo Educativo de Atención y Acompañamiento Integral para niños y adolescentes entre 8 y 18 años de edad que han sufrido maltrato infantil. Material y Métodos: Estudio transversal, descriptivo y observacional, realizado en el total de población del Internado Infantil Guadalupano. Los indicadores epidemiológicos utilizados fueron el CPOD, el ceod, IHOS, IPMA y la clasificación de Angle. Los exámenes bucales se realizaron con la luz del día utilizando espejos planos y sondas periodontales WHO, para su revisión con una duración en promedio de 5 a 10 minutos por escolar. El espacio fue un aula limpia y acondicionada para poder llevar a cabo esta actividad. La información se capturó en el Programa de Cómputo SPSS versión 17.0 Resultados: El promedio general de caries dental en dentición permanente fue de 5.6 y en la dentición temporal de 2.0 dientes con historia de esta enfermedad. La prevalencia de caries dental en esta población fue del $89 \%$. Conclusiones: El 86\% de esta población tiene necesidades de tratamiento en su boca.

Palabras clave: Maltrato, Población infantil, Caries dental 


\title{
Child Abuse: Dental Caries Risk
}

\author{
Tomas Caudillo Joya, PhD \\ Maria del Pilar Adriano Anaya, PhD \\ Sergio Antonio Chavarría López, EE
}

Profesores de la Facultad de Estudios Superiores Zaragoza Universidad

Nacional Autónoma de México

Pilar Alejandra Caudillo Adriano, MDP

Práctica Privada

\begin{abstract}
Objective: To perform the oral epidemiological diagnosis in school children of the Educational Model of Comprehensive Attention and Accompaniment for children and adolescents between 8 and 18 years of age who have suffered child abuse. Material and Methods: The study uses a crosssectional, descriptive, and observational study carried out on the total population of Guadalupano Infant Boarding School. The epidemiological indicators used were CPOD, ceod, IHOS, IPMA, and Angle classification. The oral exams were performed in daylight using flat mirrors and WHO periodontal probes, and it was reviewed with an average duration of 5 to 10 minutes per school. The space used in carrying out this activity is a clean and conditioned classroom. The information was captured using the SPSS Computing Program version 17.0. Results: The general average of dental caries in permanent dentition was 5.6 and in the temporary dentition of 2.0 teeth with a history of this disease. The prevalence of dental caries in this population was $89 \%$. Conclusions: $86 \%$ of this population has treatment needs in their mouth.
\end{abstract}

Keywords: Abuse, Child population, Dental caries

\section{Introducción}

El cuidado de la salud bucal en la población infantil se inicia en el hogar. Es la familia quién juega un papel fundamental como promotora de la salud, pero es en especial a la madre en su rol de cuidadora a quien se atribuye mayor responsabilidad por la salud de los niños, incluyendo la bucal (Couto et al., 2001; Reisine \& Douglas, 1998). En consecuencia, es el contexto familiar o ambiental el que ejerce una función protectora o de riesgo en la formación integral del niño, pues cualquier acto por acción u omisión 
realizado por individuos o por la sociedad en su conjunto, que implica una desprotección hacia un menor en la que se están vulnerando sus derechos básicos, como su libertad o su óptimo desarrollo, se puede convertir en maltrato hacia los menores (Arlinda, 2012). El maltrato infantil puede definirse como toda acción u omisión que conduzca a una agresión, abuso o descuido físico, psicológico, social y/o moral a un menor interfiriendo en su proceso normal de crecimiento (Wolfe, 1991).

La negligencia hacia la salud bucal de los niños es considerada maltrato (Arlinda, 2012). Más del 50\% de los casos de maltrato infantil presentan lesiones en cabeza, cuello, cara y boca. Sólo el $0.32 \%$ de los casos de maltrato y negligencia que acuden a las clínicas odontológicas son denunciados por los odontólogos (Arlinda, 2012). Los signos del maltrato en cara y cuello son dientes fracturados, dientes no vitales u oscurecidos, contusiones en labios o laceración del frenillo labial, fracturas del maxilar y/o mandíbula, marcas de estrangulamiento, alopecias traumáticas por tracción, hemorragia o desviación del hueso nasal, pero sin olvidar que el diagnóstico diferencial es muy importante (Arlinda, 2012). Dentro de la negligencia dental se pueden incluir la caries rampante, enfermedades periodontales, lesiones con dolor, infección y pérdida de función(Arlinda, 2012). En este sentido, la caries dental (Adriano \& Caudillo, 2015) es la principal causa de morbilidad bucal y es un problema complejo que tiene determinación económica, política, social y cultural y no solamente biológica, razón por la cual es importante la intervención de padres y tutores para el cuidado de la salud bucal desde el nacimiento de los hijos.

Para dar cumplimiento al compromiso social que tiene la Facultad de Estudios Superiores Zaragoza UNAM, sobre todo con la población vulnerable y de alto riesgo, en el 2017 se implementa un Modelo Estomatológico de Práctica Profesional Integral, modelo donde se articula la formación de profesionales en odontología, la producción de servicios y la producción de conocimientos. Estos elementos sustentan a la práctica profesional en una población cautiva que ha sufrido maltrato en alguna etapa de su infancia, razón por la cual se encuentran en el Modelo Educativo de Atención y Acompañamiento Integral niños y adolescentes entre 8 y 18 años de edad del Internado Infantil Guadalupano cuyo desarrollo integral está en riesgo (Modelo Educativo de Atención y Acompañamiento Integral, 2014). Se inicia con el diagnóstico bucal en los escolares de esta institución educativa ubicada en la Alcaldía Iztapalapa al oriente de la Ciudad de México, y tomando como base los resultados de este diagnóstico se busca transformar su perfil epidemiológico bucal a partir de la prevención integral. 


\section{Material y Métodos}

Este estudio fue descriptivo, transversal, observacional, con una población total de 117 escolares de sexo masculino, de 8 a 18 años de edad, que viven en dicho internado. La exploración bucal fue realizada por cinco pasantes de la carrera de Cirujano Dentista de esta Facultad y dos profesores responsables del estudio que fueron entrenados y calibrados en el conocimiento y aplicación de los códigos y criterios del índice CPOD. Con el fin de que la concordancia no se atribuyera al azar, se aplicó el coeficiente de KAPPA intra e inter examinadores que permite medir las variaciones de las observaciones realizadas por los revisores ( $\mathrm{K}=0.84$; IC 95\% 0.43-0.81). Los criterios usados para el CPOD y el ceo, fueron los propuestos por la OMS (World Health Organization, 2013). Así mismo, se levantó el IHOS y el IPMA para identificar problemas periodontales y la clasificación de Angle para maloclusiones. Se cumplió con las barreras de protección planteadas por la Norma Oficial Mexicana para el control y manejo de infecciones bucales (NOM-087-ECOL-SSA 1- 2000; Norma Oficial Mexicana NOM-087-ECOLSSA1 2002).

La información fue capturada en el Programa de Cómputo SPSS versión 23.

\section{Bioética}

Tomando en cuenta las normas éticas de la declaración de Helsinki de 1975, el consentimiento informado lo firmó el coordinador del Área de Cuidado Integral de la salud del internado quién es la persona responsable para otorgar el permiso para la atención bucal de los escolares (Adriano \& Caudillo, 2013).

\section{Resultados}

El Internado Infantil actualmente cuenta con 117 escolares, aunque tiene un cupo para 165. Este número puede cambiar en el momento que hay bajas realizadas por los tutores o bien por necesidades de la institución del Desarrollo Infantil de la Familia (DIF) nacional y estatal, al enviar nuevos internos.

Las edades en que se encuentran más escolares son los 11, 12 y 13 años, número que va disminuyendo conforme avanza la edad.

El promedio más elevado de caries dental es a los 17 años con $9.3 \pm 10.6$ y el menor a los 9 con $2.6 \pm 1.3$ dientes con historia de la enfermedad.

Al asociar la edad y los promedios de caries dental se encontró Significancia Estadística.

De los 117 escolares estudiados sólo 13 (11\%), se encontraron libres de caries dental. (Cuadro 1).

Con respecto a la caries dental en dentición temporal, se encontró este problema hasta los 14 años de edad. Del total de escolares revisados, 13 tenían 
erupcionados los permanentes sin que hubieran exfoliado los temporales, situación que se resolvió extrayendo los temporales, evitando una malposición dentaria.

En todas las edades existió presencia de placa dentobacteriana (PDB) encontrando un promedio general de 0.8 , lo que nos indica que cubre hasta un tercio de los dientes. (Cuadro 1).

\begin{tabular}{|c|c|c|c|c|c|}
\hline \multicolumn{6}{|c|}{$\begin{array}{l}\text { Cuadro } 1 . \\
\text { Promedios de caries dental, higiene bucal e inflamación, papilar, marginal o } \\
\text { adherida por edad de los escolares }\end{array}$} \\
\hline Edad & No. & CPOD & Ceod & IHOS & IPMA \\
\hline 8 & 6 & $3.5 \pm 1.6$ & 2.9 & 1.3 & 0.3 \\
\hline 9 & 12 & $2.6 \pm 1.3$ & 1.7 & 0.8 & 0.2 \\
\hline 10 & 14 & $4.2 \pm 4.2$ & 2.3 & 0.9 & 0.3 \\
\hline 11 & 22 & $4.1 \pm 4.1$ & 1.9 & 0.9 & 0.2 \\
\hline 12 & 23 & $4.8 \pm 4.0$ & 1.3 & 1.1 & 0.2 \\
\hline 13 & 21 & $4.5 \pm 4.5$ & 0.2 & 0.9 & 0.2 \\
\hline 14 & 9 & $8.0 \pm 4.4$ & 0.8 & 0.9 & 0.0 \\
\hline 15 & 3 & $3.3 \pm 0.5$ & 0.0 & 0.5 & 0.5 \\
\hline 16 & 2 & $3.5 \pm 0.7$ & 0.0 & 0.5 & 1.0 \\
\hline 17 & 3 & $9.3 \pm 10.6$ & 0.0 & 1.0 & 0.5 \\
\hline 18 & 2 & $13.5 \pm 3.5$ & 0.0 & 0.5 & 0.3 \\
\hline Total & 117 & $\mathrm{X}^{2}=0.015$ & $X^{2}=0.000$ & $\mathrm{X}^{2}=0.316$ & $\mathrm{X}^{2}=0.573$ \\
\hline \multicolumn{5}{|c|}{13 escolares libres de caries dental } & \\
\hline
\end{tabular}

El 27.4\% de esta población presentó maloclusiones y el 13\% presentó lesiones en los tejidos blandos que no comprometen la vida de los escolares, ya que fueron traumatismos por mordeduras en carrillos y labios (Cuadro 2).

\begin{tabular}{|l|l|l|}
\hline $\begin{array}{l}\text { Cuadro 2. } \\
\text { Distribución de Maloclusiones de Angle en los escolares }\end{array}$ \\
\hline Angle & No. & \% \\
\hline Clase 1 & 85 & 72.6 \\
\hline Clase 2 & 23 & 19.7 \\
\hline Clase 3 & 9 & 7.7 \\
\hline Total & 117 & 100.0 \\
\hline LTB = 13\% traumatismos por mordeduras en carrillos y labios \\
\hline
\end{tabular}

El nivel de severidad de 4.5 a 6.5 dientes cariados en promedio es clasificado por la OMS como alto. En este rubro se encuentra el CPOD global de los escolares con 5.6 dientes con historia de la enfermedad. El ceod fue de 2.0 y se clasifica como bajo (Organización Mundial de la Salud, 1997).

Según Greene (Ibarra et al., 2017) de 0.1 a 1.2 en promedio es buena higiene y en este rubro se encuentra el Índice de Higiene Oral (IHOS) global de los escolares de 0.8 . 
Un indice de fluorosis de 0.2 es bajo y no constituye ningún problema de salud pública.

Con respecto al ISC la meta de la OMS para el 2015 fue de 3.0 a los doce años. En esta población se encuentra muy por arriba ya que es de 7.8 dientes cariados, 4.8 dientes más de lo esperado.

Las necesidades de tratamiento son del 86\% (Cuadro 4) con una prevalencia de caries dental del $89 \%$.

\begin{tabular}{|l|l|l|l|l|l|l|l|l|l|l|}
\hline Cuadro 4. Perfil epidemiológico bucal de los escolares \\
\hline CPOD & ceod & IHOS & Cálculo & $\begin{array}{l}\text { IPM } \\
\text { A }\end{array}$ & $\begin{array}{l}\text { IF } \\
\text { C }\end{array}$ & Angle & LTB & $\begin{array}{l}\text { ISC } \\
\text { Global }\end{array}$ & $\begin{array}{l}\text { ISC } \\
\mathbf{1 2} \text { años }\end{array}$ & INT \\
\hline 5.6 & 2.0 & 0.8 & 0.09 & 0.3 & 0.2 & $27 \%$ & $13 \%$ & 9.6 & 7.8 & $86 \%$ \\
\hline 13 escolares presentan doble fila de dientes \\
89\% Prevalencia de caries dental
\end{tabular}

El motivo de ingreso al internado se obtuvo de los expedientes de los escolares. Independientemente de éstos, los promedios de caries dental son elevados, lo que demuestra que es un reflejo de la casi nula atención de los padres o tutores hacia los niños, que los hace ser más vulnerables a los problemas de su salud bucal.

Cabe resaltar que los promedios más elevados de caries dental se encontraron en los niños con situación de calle, con 9.7 dientes con historia de la enfermedad (Cuadro 5).

El comportamiento de la caries dental en la dentición temporal es diferente con respecto a los motivos de ingreso; esto se puede deber a que en cada una de las opciones se encontraron niños y adolescentes con o sin dientes temporales (Cuadro 5).

\begin{tabular}{|l|l|l|l|}
\hline \multicolumn{4}{|l|}{ Cuadro 5. Promedios de caries dental de los escolares por motivo de ingreso al internado } \\
\hline Motivo de ingreso & No. & CPOD & ceod \\
\hline Extravío & 3 & $3.0 \pm 4.3$ & $2.3 \pm 1.5$ \\
\hline Pobreza extrema & 17 & $4.2 \pm 3.7$ & $1.1 \pm 1.9$ \\
\hline Violencia familiar & 3 & $7.3 \pm 8.3$ & 0.0 \\
\hline Problemas de conducta & 23 & $4.2 \pm 4.0$ & $1.2 \pm 1.4$ \\
\hline Abandonado por los padres & 9 & $4.1 \pm 5.2$ & $1.0 \pm 2.0$ \\
\hline Desintegración familiar & 8 & $2.6 \pm 1.4$ & $1.9 \pm 2.2$ \\
\hline Casa cuna & 2 & $6.5 \pm 2.1$ & $3.0 \pm 4.2$ \\
\hline Maltrato & 28 & $5.7 \pm 4.0$ & $2.1 \pm 1.6$ \\
\hline Familia disfuncional & 11 & $5.9 \pm 6.1$ & $1.1 \pm 1.3$ \\
\hline Madre enferma terminal & 2 & 13.00 & 0.0 \\
\hline Situación de calle & 3 & $9.7 \pm 2.8$ & $0.7 \pm 1.1$ \\
\hline Orfandad & 7 & $3.3 \pm 4.1$ & $0.3 \pm 0.7$ \\
\hline Padre con cáncer terminal & 1 & 2.00 & 8.0 \\
\hline & & $\mathrm{p}=0.469$ & $\mathrm{p}=0.060$ \\
\hline
\end{tabular}


Tradicionalmente como parte de la cultura popular son las madres quienes se encargan del cuidado de los hijos y, en consecuencia, de transmitir los hábitos de higiene, en este caso del cuidado de la boca. En vista de la situación particular de las madres de los niños y adolescentes que se encuentran en el internado, se observa la vulnerabilidad de estos, debido a que los promedios más elevados de caries dental los presentan los escolares con madres que los abandonaron, con problemas psiquiátricos o drogadictas. Así mismo, al padecer las madres enfermedades terminales estas descuidan a los hijos por esta situación y esto los hace más vulnerables (Cuadro 6).

Es importante considerar que no se encontró el mismo número de niños, en cada una de las opciones de situación de la madre, lo que hace que existan promedios muy elevados como en las enfermas terminales donde hay dos escolares con un promedio de caries dental de 13.5 dientes afectados por este problema. En este sentido, se encontraron diferencias significativas entre los promedios de caries dental en dentición permanente de los escolares y la situación de las madres (Cuadro 6).

\begin{tabular}{|l|l|l|l|}
\hline \multicolumn{4}{|c|}{ Cuadro 6. Promedios de caries dental de los escolares por situación de las madres } \\
\hline Situación de la madre & No. & CPOD & ceod \\
\hline Empleadas & 30 & $5.5 \pm 4.5$ & $1.3 \pm 1.6$ \\
\hline Fallecidas & 7 & $2.1 \pm 1.7$ & $0.3 \pm 0.7$ \\
\hline Abandonaron a sus hijos & 22 & $10.2 \pm 5.8$ & $1.2 \pm 2.1$ \\
\hline Alcohólicas & 8 & $4.4 \pm 3.5$ & $1.0 \pm 1.5$ \\
\hline Empleadas Domésticas & 10 & $3.1 \pm 2.6$ & $1.3 \pm 2.0$ \\
\hline Se dedican al hogar & 3 & $3.7 \pm 4.0$ & $0.7 \pm 1.1$ \\
\hline Se encuentran en la cárcel & 6 & $3.0 \pm 1.5$ & $2.5 \pm 3.0$ \\
\hline Comerciantes & 18 & $3.1 \pm 2.6$ & $1.2 \pm 1.5$ \\
\hline Con problemas psiquiátricos & 5 & $5.2 \pm 1.0$ & $1.6 \pm 3.0$ \\
\hline Drogadictas & 3 & $5.3 \pm 5.8$ & $2.0 \pm 1.7$ \\
\hline Alcohólica y drogadicta & 2 & $4.5 \pm 2.1$ & $0.5 \pm 0.7$ \\
\hline Desempleadas & 1 & 3.0 & 0.0 \\
\hline Enfermas terminales & 2 & $13.5 \pm 0.7$ & 0.0 \\
\hline & & $\mathrm{p}=0.010$ & $\mathrm{p}=0.860$ \\
\hline
\end{tabular}

Con respecto a la situación de los padres, el $49 \%$ abandonó el hogar, para el 30\% su situación es de algún problema social, ya sea alcoholismo, drogadicción o privado del libertad; el $21 \%$ se encuentra finado o bien realiza alguna actividad social productiva (albañil, comerciante, empleado). Sin embargo, en lo que respecta a los promedios de caries dental en dentición permanente todas las opciones son similares, no encontrando diferencias entre ellos (Cuadro 7). 


\begin{tabular}{|l|l|l|l|}
\hline $\begin{array}{l}\text { Cuadro 7. } \\
\text { Promedios de caries dental de los escolares por situación del padre }\end{array}$ \\
\hline Situación del padre & No. & CPOD & ceod \\
& & & \\
\hline Abandonó a la familia & 57 & $5.0 \pm 4.5$ & $1.0 \pm 1.7$ \\
\hline Finado & 10 & $4.3 \pm 5.0$ & $0.3 \pm 0.6$ \\
\hline Cárcel & 13 & $5.0 \pm 4.2$ & $1.5 \pm 1.5$ \\
\hline Alcohólico y violento & 6 & $4.3 \pm 2.3$ & $1.2 \pm 0.9$ \\
\hline Albañil & 4 & $7.0 \pm 9.3$ & $0.7 \pm 0.9$ \\
\hline Enfermedad terminal & 1 & 8.0 & 3.0 \\
\hline Empleado & 9 & $5.2 \pm 3.5$ & $1.6 \pm 2.3$ \\
\hline Alcohólico, Drogadicto & 16 & $6.0 \pm 4-6$ & $1.0 \pm 0.5$ \\
\hline Comerciante & 1 & 3.00 & 6.0 \\
\hline & & $\mathrm{P}=0.867$ & $\mathrm{P}=0.001$ \\
\hline
\end{tabular}

La procedencia de los niños con respecto a la Ciudad de México corresponde a las Alcaldías políticas consideradas con bajo desarrollo social como son Iztapalapa y Tláhuac.

Del Estado de México, la mayor procedencia es: Ciudad Nezahualcóyotl, Chalco, Chimalhuacán y Ecatepec.

Del interior de la República son pocos los niños que se encuentran en el internado.

\begin{tabular}{|c|c|c|c|}
\hline \multicolumn{4}{|c|}{$\begin{array}{l}\text { Cuadro 8. } \\
\text { Promedios de caries dental de los escolares por zona de procedencia }\end{array}$} \\
\hline Domicilio & No. & ceo & CPOD \\
\hline DIF Nacional & 14 & $0.3 \pm 0.6$ & $3.7 \pm 4.3$ \\
\hline \multicolumn{4}{|l|}{ Ciudad de México } \\
\hline Álvaro Obregón & 1 & 3 & 16.0 \\
\hline Miguel Hidalgo & 1 & 6 & 5.0 \\
\hline Iztapalapa & 25 & $1.1 \pm 1-3$ & $4.3 \pm 4.6$ \\
\hline Coyoacán & 5 & $1.0 \pm 1.7$ & $6.0 \pm 6.1$ \\
\hline Xochimilco & 4 & 0 & $7.0 \pm 5.7$ \\
\hline Tláhuac & 8 & $2.6 \pm 2.5$ & $4.0 \pm 1.9$ \\
\hline Venustiano Carranza & 2 & 0 & $3.5 \pm 2.1$ \\
\hline Milpa Alta & 1 & 8 & 2.0 \\
\hline Gustavo A. Madero & 1 & 0 & 3.0 \\
\hline Cuajimalpa & 1 & 2 & 1.0 \\
\hline Tlalpan & 2 & $1.0 \pm 1.4$ & $10.5 \pm 5.0$ \\
\hline \multicolumn{4}{|l|}{ Estado de México } \\
\hline Tlalnepantla & 5 & $0.8 \pm 1.3$ & $4.4 \pm 2.6$ \\
\hline Nezahualcóyotl & 8 & $0.9 \pm 2.1$ & $4.6 \pm 4.9$ \\
\hline Los Reyes & 3 & $1.7 \pm 1.5$ & $2.3 \pm 2.0$ \\
\hline Ixtapaluca & 2 & 0.0 & $1.0 \pm 1.4$ \\
\hline Chicoloapan & 2 & 0.0 & $3.5 \pm 0.7$ \\
\hline Huehuetoca & 1 & 2.0 & 4.0 \\
\hline Chimalhuacán & 6 & $1.2 \pm 1.8$ & $5.8 \pm 4.6$ \\
\hline
\end{tabular}




\begin{tabular}{|l|l|l|l|}
\hline Ecatepec & 7 & $1.7 \pm 2.2$ & $2.4 \pm 1.9$ \\
\hline Nicolás Romero & 1 & 0.0 & 21.0 \\
\hline Chalco & 9 & $2.0 \pm 2.5$ & $5.1 \pm 3.8$ \\
\hline Huixquilucan & 1 & 4.0 & 6.0 \\
\hline Naucalpan & 2 & 0.0 & $8.0 \pm 5.6$ \\
\hline Provincia & 2 & $1.0 \pm 1.4$ & $7.0 \pm 1.4$ \\
\hline Puebla & 2 & 0.0 & 0.0 \\
\hline Veracruz & 1 & 1.0 & 11.0 \\
\hline Guerrero & 1 & 0 & 2.0 \\
\hline Pachuca & 1 & $\mathrm{p}=0.013$ & $\mathrm{p}=0.040$ \\
\hline & &
\end{tabular}

\section{Discusión}

Realizar diagnósticos del Proceso Salud Enfermedad del sistema estomatognático en la población infantil y adolescente en contextos específicos hace posible identificar las condiciones de la salud bucal en grupos de población en una realidad específica, donde las acciones que se realicen deben ser las que correspondan a las necesidades identificadas, de tal manera que puedan ser efectivas, eficaces, y al alcance de la población en estudio.

Los niños que ingresan al Internado Infantil Guadalupano lo hacen porque se ha identificado que su desarrollo integral está en riesgo (Modelo Educativo de Atención y Acompañamiento Integral, 2014), ya sea por orfandad, abandono, extravío, bajos recursos económicos, desintegración familiar, violencia, abuso, maltrato o explotación, o bien por la experiencia de calle, rezago o deserción escolar y sin primaria concluida (Modelo Educativo de Atención y Acompañamiento Integral, 2014).

La situación anteriormente descrita hace a la población escolar vulnerable a su salud en general y odontológica en particular. Así lo refleja el promedio general de caries dental de 5.6 dientes con historia de la enfermedad, que de acuerdo con la OMS es considerado como de altoriesgo (Piovano, Squassi \& Bordoni, 2010). Así mismo el índice de caries significante es de 7.8 dientes afectados, 4.8 dientes más de lo esperado por la OMS que establece que para el 2015 a los doce años debe ser de 3.0 (Organización Mundial de la Salud, 1997).

La prevalencia de caries dental fue del $89 \%$, es decir nueve de cada diez escolares presenta este problema, lo que da como resultado el elevado índice de necesidades de tratamiento.

Ante la magnitud de estos problemas es necesario actuar con medidas de prevención integral, de tal manera que se evite la agudización de éstos problemas y con ello la complicación de los mismos y evitar a futuro la pérdida de los dientes. 
Como se observa en este grupo de población los promedios de caries dental son muy diferentes a los reportados por la Encuesta Nacional de Caries Dental (2001), donde el promedio de dientes cariados de 6 a 12 años en la Ciudad de México fue de 1.2 dientes con historia de la enfermedad. Así mismo el promedio de caries dental encontrado en las Alcaldías de Milpa Alta fue 3.4 (Adriano \& Caudillo,2007), y en Iztapalapa con 3.0 dientes con historia de esta enfermedad (Adriano \& Caudillo, 2018), lo hace totalmente diferente a lo encontrado en el internado.

Con respecto a los problemas periodontales, se circunscriben a inflamación papilar, reflejo de que la placa dentobacteriana se encuentra cubriendo hasta un tercio del diente. Las maloclusiones en los escolares en el momento actual se encuentran en un proceso de diagnóstico clínico para identificar su severidad e iniciar con los tratamientos ortodóncicos necesarios. En el momento del estudio epidemiológico bucal se encontraron 13 escolares con dentición temporal y permanente en los mismos espacios, lo que obligó a la extracción de todos los dientes temporales que ocasionaban doble hilera de dientes, previniendo así daños mayores de maloclusiones en el corto y mediano plazo.

Las lesiones en los tejidos blandos no representan un problema grave en los escolares, ya que se identificaron lesiones producto de mordeduras y traumatismos, sin mayores consecuencias.

Todos los escolares que se encuentran en el internado son remitidos del DIF nacional, Estatal o diferentes instituciones altruistas. El diagnóstico encontrado de su salud bucal es el reflejo de las condiciones de vida en las que se están desarrollando estos niños. Muchos de ellos nunca han sabido lo que es tener un papá o una mamá ya que desde que nacieron se encuentran en estas instituciones donde su familia la va a conformar sus compañeros, maestros y trabajadores que allí se encuentran.

Con respecto a los niños que si han nacido en un núcleo familiar, este se encuentra desintegrado, es disfuncional, o son abandonados, extraviados o migrantes, con el padre o la madre metidos en las drogas, alcohol, violencia, abuso de todo tipo, lo que los hace ser vulnerables en su salud mental, emocional y física, con niveles de salud en general y bucal en particular precarios.

Las zonas de donde proceden estos niños son: Ciudad de México, al igual que de la Alcaldía Iztapalapa, considerada como marginada por sus indicadores socioeconómicos manejados por el INEGI, así como también de las más violentas Hay 25 niños des esta área en el internado.

Del Estado de México 17 escolares proceden de los municipios de Nezahualcóyotl y Chalco, municipios considerados con las mismas características socioeconómicas que Iztapalapa. Del interior de la república mexicana sólo se encuentran 5 escolares. 
Al momento de incorporarse la Facultad de Estudios Superiores Zaragoza UNAM para prestar sus servicios odontológicos al internado, esta cumple con uno de los principios que la caracterizan, especialmente la carrera de Cirujano Dentista con sus programas del área de conocimiento de Odontología Social, de formar a sus alumnos en diferentes contextos sociales y con ello el alto compromiso social con los grupos más necesitados y vulnerados del país. Esto también hace posible la implementación de diferentes modelos estomatológicos de práctica profesional integral que respondan a los cambios permanentes que vive la sociedad, y con ello enfatizar el espíritu humanitario que debe prevalecer en los egresados implementando un quehacer profesional, más justo, igualitario, comprometido, equitativo, que cumpla con las expectativas que la sociedad reclama de esa profesión. Es por ello que a partir de este diagnóstico se implementa un proyecto de intervención de prevención integral con el propósito de transformar el perfil epidemiológico bucal de los niños tratados.

\section{Conclusiones}

Los resultados del perfil epidemiológico bucal que se obtuvieron de los escolares del Internado Infantil Guadalupano, demuestran por su magnitud que son un problema de salud pública y en consecuencia el $86 \%$ de los escolares necesita algún tipo de tratamiento bucal.

Los escolares que viven en el Internado Infantil Guadalupano son el reflejo de las condiciones sociales, políticas y económicas generales que caracterizan a la sociedad actual, las cuales determinan la calidad y los estilos de vida de los grupos sociales específicos; en el caso particular de los escolares dónde nacen, viven y se desarrollan y cómo los grupos sociales son permeados por su contexto. La salud en general y odontológica en particular, es también un reflejo de las condiciones anteriormente citadas.

\section{References:}

1. Adriano, M. \& Caudillo, T. (2007). Diagnóstico del proceso salud enfermedad estomatológico en la población escolar de la delegación Milpa Alta. Edit. FES-Zaragoza UNAM. México 2007; pp. 203-218.

2. Adriano, P. \& Caudillo, T. (2013). Valores éticos en Odontología. Rev. Odontología actual México; 10(124): 58-60.

3. Adriano, P. \& Caudillo, T. (2015). Complejidad del Proceso Salud Enfermedad Estomatológico. Edit. Facultad de Estudios Superiores Zaragoza UNAM México; 74-75.

4. Adriano, M. \& Caudillo, T. (2018). Diagnóstico de salud bucal en la población escolar de seis a doce años de la delegación Iztapalapa. Rev. Odontopediatría Act. México 2015; 4(13): 32-38. 
5. Arlinda, L. (2012). Manejo y diagnóstico del niño/a víctima del maltrato infantil en la clínica dental. Universidad Cardenal Herrera. España.

6. Couto, CMMM., Rio, LMSP., Martins, RD., Martins, CC. \& Paiva, SM. (2001). A percepção de mães pertencentes a diferentes níveis socioeconómicos sobre a saúde bucal dos seus filhos bebés. Arq Odontol; 37(2): 121-132.

7. Encuesta Nacional de Caries Dental (2001). Secretaría de Salud. México.

8. Ibarra, K., Calle, M., López, E., \& Heredia, D. (2017). Índice de higiene oral comunitario en escolares de 12 años. Rev. Evid. Odontol. Clinic. Ecuador: 3(2): 46-50

9. Modelo Educativo de Atención y Acompañamiento Integral (2014). Internado Infantil Guadalupano A.C. La Salle. Edic. Benjamín Franklin. México; pp.14-60.

10. Norma Oficial Mexicana NOM-087-ECOL-SSA1-2002. Protección ambiental-Salud ambiental-Residuos peligrosos biológicoinfecciosos-Clasificación y especificaciones de manejo. [Monografía en internet]. [Consultado 2018 enero 12]. Disponible en: http://www.salud.gob.mx/unidades/cdi/nom/087ecolssa.html.

11. Organización Mundial de la Salud (1997). Encuestas básicas de salud bucodental. Métodos básicos. $4^{\mathrm{a}}$ ed. Ginebra; 1-27.

12. Piovano, S., Squassi, A., \& Bordoni, N. (2010). Estado del arte de indicadores para la medición de caries dental. Cátedra de Odontología Preventiva y Comunitaria, Facultad de Odontología, Universidad de Buenos Aires. Revista de la Facultad de Odontología (UBA) Argentina; 25: 30-38.

13. Reisine, S. \& Douglas, JM. (1998). Psychosocial and Behavioral Issues in ECC. Community Dent Oral Epidemiol. 26(Supl. 1): 32-44.

14. Wolfe, D. (1991). "Programa de conducción de niños maltratados". Ed. Trillas. México, DF.

15. Santana Tavira R. Sánchez Ahedo R. Herrera Bastos E. (1998). "El maltrato infantil: un problema mundial" Salud Pública de México; 40(1): 58-65.

16. World Health Organization (2013). Oral health surveys: basic methods. Fifth Edition. 